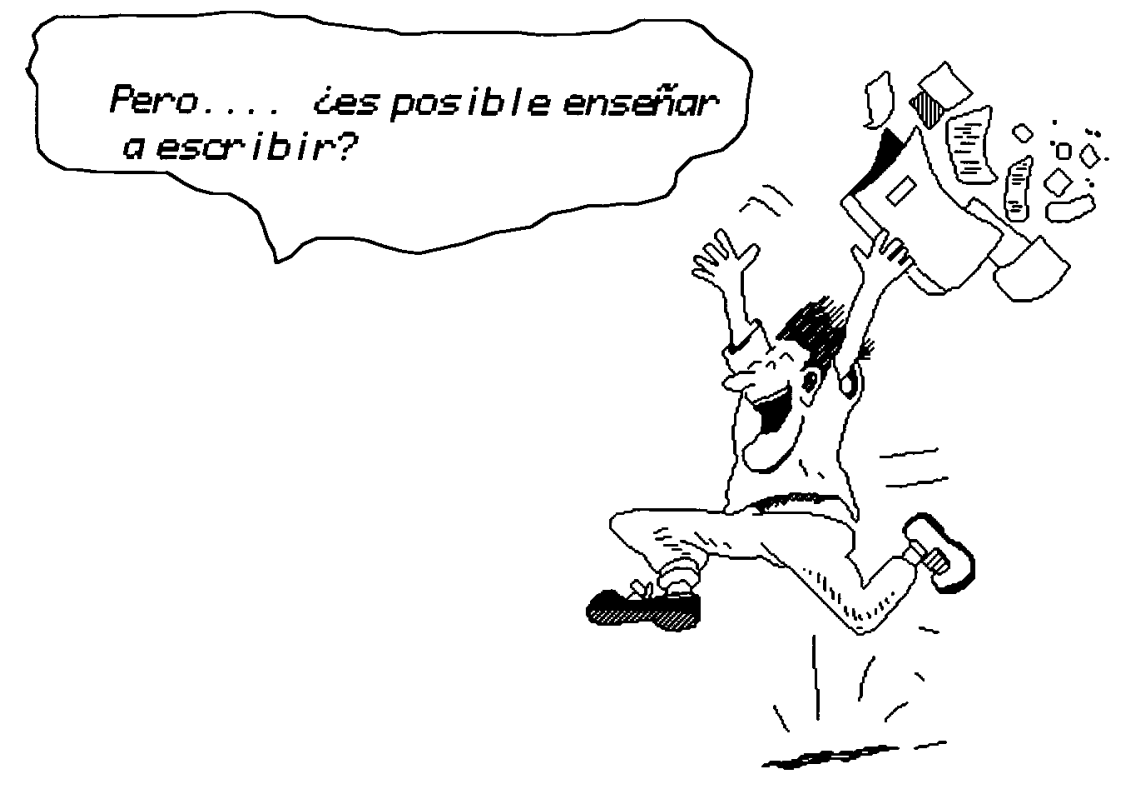




\title{
Leer y escribir: ¿cómo lograrlo desde la perspectiva del lenguaje integrado?
}

\author{
Pilar Lacasa, José Jerónimo Anula y Beatriz Martín
}
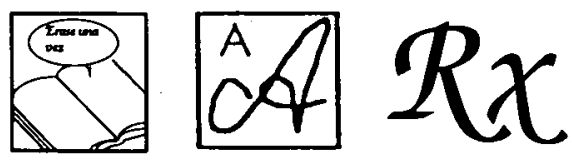

El enfoque por tareas en la enseñanza de la lengua extranjera ba recibido una gran atención en los últimos años. Sin embargo, existen pocos trabajos que, desde la propia práctica educativa, reflexionen sobre sus ventajas $e$ inconvenientes. La experiencia que presentan los autores en este artículo llena esta laguna.

\section{EL NIÑO ANTE LO IMPRESO}

Los niños de las sociedades Occidentales viven inmersos en el medio impreso. Las «letras» forman parte de su vida cotidiana, aunque el modo de aproximarse a ellas es, sin duda ninguna, múltiple. Lograr que ese universo impreso sea significativo para ellos es uno de los objetivos más importantes del lenguaje integrado. En cualquier caso, hemos de tener presente que el lenguaje oral, de acuerdo con este enfoque, es el puente desde el que acceder al lenguaje escrito.

Newman (1985b), siguiendo muy de cerca los presupuestos de esta perspectiva, parte de cómo los niños aprenden a utilizar el lenguaje oral en situaciones naturales, especialmente en la familia, para determinar estrategias que puedan aplicarse en la escuela. Los niños, nos dice, aprenden a utilizar el lenguaje participando en las conversaciones. Utilizando cualquier recurso lingüístico que tienen a su alcance llegan a dominar tanto el conocimiento del mundo, el significado, como las formas sintácticas y semánticas a través de las cuales se construye ese significado. Pero nada de esto será posible si los niños no se desenvuelven en un contexto comunicativo en el que interactúan con otros niños y con los adultos.

Quienes comparten la filosofía del lenguaje integrado insisten, por ejemplo, en cómo los padres se preocupan, sobre todo, por lograr una comunicación adecuada más que por enseñar a sus hijos a hablar. Para que el niño logre entender el significado los padres han de interpretar la situación y conocer tanto los intereses, deseos y necesidades infantiles como las habilidades linguísticas de sus hijos. Utilizan cualquier pista que el niño les pueda aportar y, desde aquí, le proporcionan la información que necesita para lograr comprender plenamente la situación. De esta forma le ayudan a entender qué aspectos de la situación son los realmente importantes. Las contribuciones del adulto son fuentes desde las que el niño amplía sus recursos comunicativos, su comprensión del mundo y el modo en que éste opera. Además, en 
un entorno comunicativo, los niños reciben información sobre lo que dicen. Se dan cuenta de si sus expresiones, tanto en un nivel interpersonal como conceptual, han sido comprendidas. Descubren el efecto interactivo de un enunciado a través de confirmaciones o ampliaciones de lo que ellos mismos han dicho. En suma, por medio de la conversación se les muestra el lenguaje en acción.

¿Pero cuáles son realmente las diferencias en la forma en que padres y maestros utilizan el lenguaje? Los padres crean de forma espontánea un entorno en el que los niños participan como compañeros conversacionales, se les permite preguntar y hacer comentarios sobre cualquier cosa y gran parte de las conversaciones tienen lugar mientras realizan actividades cotidianas. Los padres responden significativamente al lenguaje infantil en función de las demandas de la situación. De todo ello los maestros pueden aprender diversas estrategias que facilitarían el aprendizaje de los niños (Newman, 1985b). Será necesario, por ejemplo, que aprendan a descubrir las intenciones del niño, algo que puede ser más difícil duda más difícil que en el caso de los pares, y que le ayuden a encontrar una forma de decir lo que quiere. También los maestros han de crear situaciones en las que los niños puedan aprender a partir de intercambios en los que únicamente son participantes periféricos.

En síntesis, de acuerdo con Newman (1985a) el contexto social del aprendizaje es importantes y relevante si queremos favorecer en el niño el desarrollo de aspectos relacionados con la lecto-escritura. Enseñar a leer y escribir será más fácil si consideramos cómo los niños aprenden a utilizar el lenguaje oral en multitud de contextos. Leer y escribir en entornos significativos contribuirá a facilitar el conocimiento y las estrategias necesarias para lograr una lecto-escritura fluida.

\section{ACERCAR AL NIÑO A LA ESCRITURA}

Hemos señalado ya en uno de los artículos incluidos en este volumen que para los autores que trabajan desde la perspectiva del lenguaje integrado la escucha, la palabra, la lectura y la escritura son actividades entre las que existe una profunda interacción. Aceptar este presupuesto lleva consigo importantes implicaciones educativas que suponen, por una parte, un intento de ofrecer una alternativa a los enfoques más clásicos en la enseñanza de la lecto-escritura y, por otra parte, aceptar que los niños aprenden a leer y escribir de un modo similar a como aprenden el lenguaje oral, es decir, sin necesidad de que exista una enseñanza explícita y directa hacia un conjunto de destrezas que lo hagan posible.

Nos detendremos un momento en un ejemplo. Mónica y Enrique son dos niños de seis años que están comenzando a escribir. Para ellos la escritura tiene un significado funcional $y$ es una actividad que llevan a cabo de forma significativa, hasta tal punto que llegan a introducirla en sus juegos. Un día organizan una pequeña «venta» y es necesario señalar los precios de cada objeto que han ido recogiendo en sus casas y que quieren vender. Creemos que pretenden tanto recordar los precios ellos mismos cómo mostrarlos a sus «potenciales clientes». La «lista de cosas» que compone Mónica (figura 1) va a utilizar, en principio, un lenguaje que mezclará los dibujos y los números, pero la niña escribe también en la otra cara de la hoja un título: «lista de cosas". Sólo más tarde, cuando uno de nosotros intenta descifrar «la lista» con los niños, Enrique anotará el nombre de cada uno de los objetos que querían vender; lo logró con una pequeña ayuda de adulto (figura 2). Pero podemos preguntarnos qué significado tendrá esta situación si la interpretamos desde la filosofía del lenguaje integrado. Afirmaremos, en primer lugar, que la escritura es para los niños algo realmente significativo, tanto que han llegado a introducirla progresiva y espontáneamente en sus juegos. Podemos ir todavía más lejos y pensar que los niños 
33

conocen bien que sus palabras, expresadas tanto oral como gráficamente, pueden corresponder a objetos que encuentran en situaciones cotidianas. Observamos, además, que son capaces de manejar, conjuntamente, distintos sistemas de expresión: introducen los objetos en su «lista de cosas» por medio de dibujos aunque, realmente, sabían utilizar "la escritura de los adultos» que sin duda, en ese momento, les planteaba mayores dificultades y era además menos expresiva. Finalmente, el uso de la escritura surgió en un contexto social, por una parte, los niños querían comunicarse con «sus posibles clientes", por otra, juntos elaboraron «la lista».

FiguRA 1

Mónica y Enrique escriben espontáneamente mientras juegan

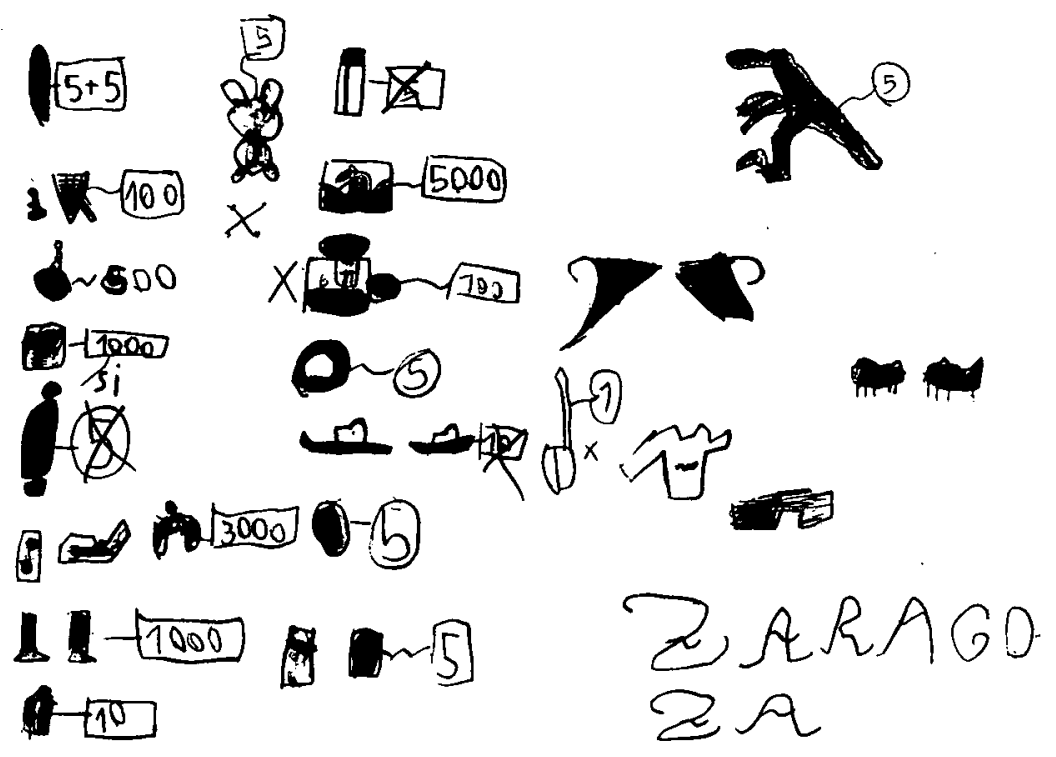

FIGURA 2

Enrique "descifra» con el adulto su dibujo

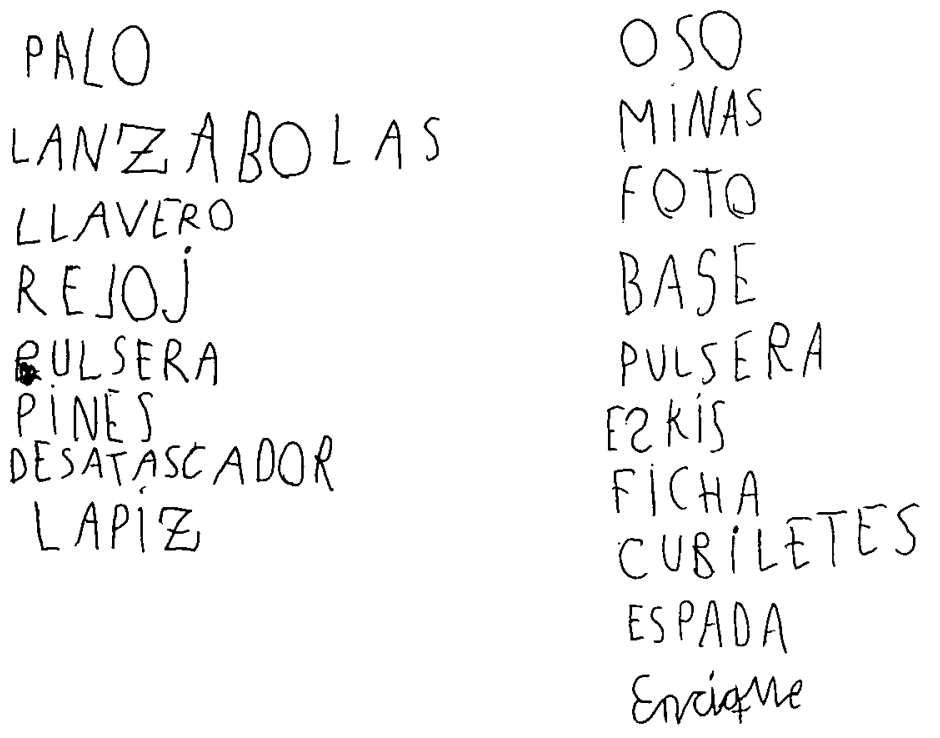




\section{EL PUNTO DE PARTIDA}

Tras los programas tradicionales de enseñanza de la lengua escrita parece esconderse la idea de que los niños, antes de ser capaces de componer un texto, necesitan dominar un conjunto de habilidades, organizadas incluso jerárquicamente. Especial importancia entre ellas tendrá la capacidad de dominar el trazo e, incluso, copiar la letras del alfabeto, algo que es anterior a la posibilidad de utilizar símbolos para transcribir los sonidos del lenguaje. Desde esa mima perspectiva, y desde el momento en que los niños comienzan a controlar sus habilidades gráficas, la ortografía se convierte en la preocupación dominante del profesor de forma que los niños han de llegar a transcribir y dominar correctamente listas de palabras que podrían considerarse un vocabulario básico. El próximo paso será transcribir o copiar textos, poniendo especial acento en destrezas tales como la ortografía, puntualización o diseño de letras mayúsculas. En suma, los aspectos mecánicos, los rasgos superficiales, son todavía considerados en algunos programas de escritura y en ciertos libros de texto como aspectos fundamentales que los niños ban de llegar a dominar antes de poder utilizar la lenguaje escrita atribuyéndole un significado.

No es nuevo decir que numerosos maestros tratan de buscar caminos diferentes cuando se trata de enseñar a escribir a los niños. A modo de síntesis nos fijaremos ahora en los aspectos que pueden considerarse los presupuestos del enfoque del lenguaje integrado.

- En primer lugar la escritura se asocia a la idea de "autor», es decir, quién escribe quiere expresar o comunicar algo. La escritura es un medio de expresar el pensamiento aunque éste sufra determinadas transformaciones. El escritor escribe lo que piensa, aunque su pensamiento sea mucho más rico que aquello que puede ser capturado por el lápiz.

- En segundo lugar, el escritor escribe en función de unas determinadas metas. Por ello, aunque el escritor necesita poner en práctica ciertas habilidades de carácter general, su atención se centrará en aspectos específicos en función de sus metas.

- En tercer lugar, la aproximación del lenguaje integrado insiste en que los niños en los primeros niveles escolares han de aproximarse a la escritura como a un juego, posteriormente irán dominando ciertas convenciones de forma que puedan satisfacer audiencias determinadas.

- Finalmente, el enfoque del lenguaje integrado propone que los niños «escriben para aprender»y no tanto que «aprenden a escribir». Se busca, la función más que la forma ya que, seguramente, se piensa que la forma sigue a la función.

Pero, ¿cuáles son los trabajos en los que se inspiran este enfoque? Especial significado, por ejemplo, adquieren las aportaciones de Graves (1983), que insistió en la interacción que existe entre diferentes formas de expresión simbólica, más concretamente, el dibujo, el lenguaje oral y la escritura. Es decir, hablar podía conducir a dibujar, dibujar a escribir o hablar, etc., y ello porque existe una estrecha interacción entre todos ellos. En este contexto se recalca la idea de que los escritores encuentran el significado de lo que escriben mientras producen el texto, generándose así un continuo intercambio entre el texto emergente y el pensamiento. Graves mostró también que cuando tienen lugar un desarrollo en determinados aspectos del lenguaje escrito, por ejemplo, la sintaxis, la puntuación, el significado, etc. pueden producirse retrocesos en aspectos que los niños ya habrían adquirido previamente. En suma, el desarrollo supone el refinamiento gradual de un todo en varios frentes a la vez, de forma que no consiste tanto en atravesar una serie de estadios como en la capacidad de utilizar progresivamente determinadas estrategias, disponibles incluso para niños 
muy pequeños. De estas ideas se derivan algunas implicaciones educativas. En este contexto se defenderá también que la forma no precede a la función sino que es ésta, orientada hacia la comunicación de ideas a uno mismo o a los otros, la que crea la motivación. Los niños aprenden sobre la escritura mientras leen y viceversa. Aprender a leer y escribir es un proceso que supone experimentar con el lenguaje. Una lectura o escritura fluidas no son un producto final, la escritura se desarrolla en múltiples direcciones a la vez y ello no siempre a través de un camino fácil. La escritura necesita mucho más soporte y apoyo comunicativo que un programa prescriptivo.

En una línea similar, Newman (1985b) señala algunas de las conclusiones a las que han llegado algunos de los autores que sirven de fundamento a los enseñantes que adoptan la perspectiva del lenguaje integrado. Considera también que el desarrollo no se produce de acuerdo con una linea progresiva y uniforme, sino que la historia instruccional, la lectura, el contexto cultural y social influyen en la complejidad de la escritura del niño. Hay que tener en cuenta, por lo tanto, que la escritura infantil está también influida por la respuesta del maestro, la interacción con los iguales o con la familia. En este sentido señala, por ejemplo, cuáles son las características de los primeros encuentros que los niños tienen con el lenguaje escrito. Se sugiere que èl desarrollo de la lecto-escritura depende de las experiencias previas que hayan tenido en el hogar. Ya en los años setenta, Clay (1975) habría mostrado que que los primeros escritos de los niños comienzan mucho antes de que reciban una enseñanza formal.

También Harste y sus colaboradores (1984) se refieren al desarrollo de las habilidades relacionadas con la lecto-escritura y ello no en términos de estadios sino de estrategias específicas y señala algunos aspectos de ese proceso que seguramente podrán orientar la actividad del profesor:

a) Los niños esperan que el lenguaje escrito tenga sentido y pueden interpretarlo en función de sus propias expectativas ante el mensaje. Newman (1985b) se refiere al siguiente ejemplo. Ante el cartel muy frecuente en los restaurantes americanos, en el que dice «Esperen a que el camarero les atienda», pero que, en esta ocasión, está situado junto a un árbol de Navidad, una niña de dos años interpreto «No tocar». Estamos ante un buen ejemplo de cómo la cómo la comprensión de la funcionalidad, e incluso la forma del lenguaje escrito, se desarrollan desde etapas muy tempranas.

b) Los niños aprovechan lo que saben sobre el lenguaje y el mundo para interpretar el lenguaje escrito. Por ejemplo, los niños son capaces de interpretar carteles o anuncios que habitualmente ven en la calle.

c) Experimentan con el lenguaje e intentan escribir, incluso sin conocer demasiado bien las reglas de este medio de expresión. Mónica, que en este caso está comenzando a aprender a manejar diversos juegos en el ordenador que utilizan los adultos, tiene que seguir una serie de pasos hasta encontrar el directorio en el que se encuentran sus juegos. De forma espontánea, ante las instrucciones del adulto, comienza a hacerse «una chuleta» en la que, en su propio lenguaje y mezclando representaciones gráficas y letras, aparecen los pasos que debe seguir.

d) Lo que los niños aprenden interactuando con el lenguaje es algo que podrá ser aplicado en ocasiones posteriores. Unos meses más tarde Mónica (figura 3) no sólo es capaz de utilizar su chuleta, útil en una situación inmediata, sino que cuando juega con el ordenador un partido de fútbol decide anotar los nombres de los jugadores de los equipos, y la posición que cada uno de ellos ocupan en el campo. La niña, además de haber ido más allá de la situación inmediata, ha ido aceptando las convenciones linguísticas. Espontáneamente ha reproducido lo que aparece en la pantalla del ordenador y, además, se sirve de la escritura para expresar lo que, en ese contexto, es más importante para ella. Anota los nombres de los jugadores, en la posición que les corresponde en el dibujo, los números que los identifican e incluso, en los márgenes del dibujo el resultado del partido 3-1 a favor del Barcelona. 


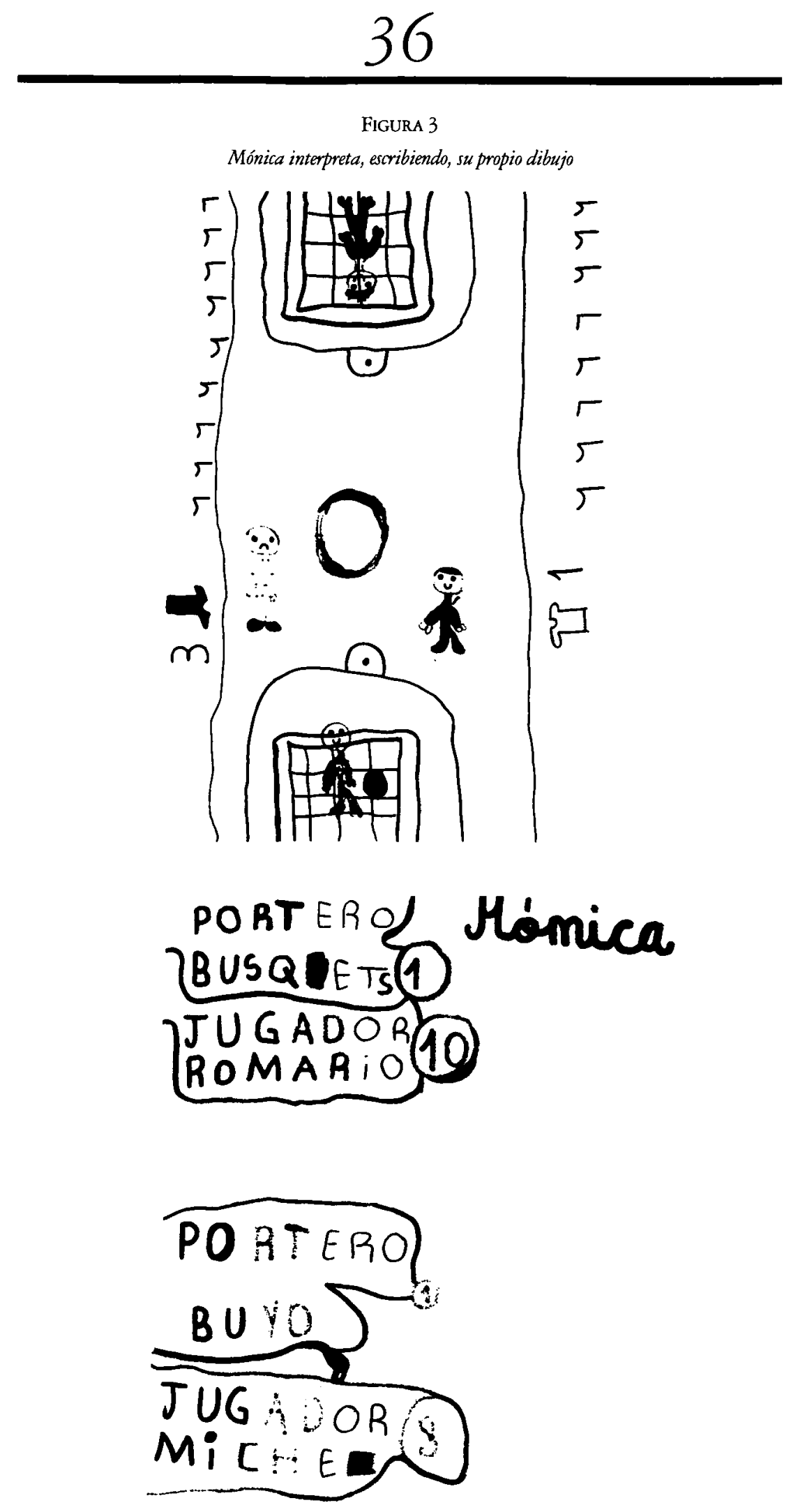




\section{ENSEÑAR A PRODUCIR «UNA ESCRITURA AUTÉNTICA»}

Hemos señalado ya que la lecto-escritura, desde la perspectiva del lenguaje integrado, ha de ser ante todo una actividad significativa y ello tanto para el lector como para el escritor. En este contexto, Edelsky y Smith (1989) introducen el concepto de «escritura auténtica» y consideran que en este tipo de expresión las diferentes dimensiones del lenguaje operan de forma interactiva e interindependiente. Por ejemplo, cuando la meta de lo escrito es facilitar el recuerdo de algo que es importante para el escritor, por ejemplo, las compras que han de realizarse en el supermercado, las consideraciones pragmáticas pueden afectar a las sintácticas. Para lograr que la lecto-escritura sea auténtica, quienes se sitúan en la perspectiva del lenguaje integrado insisten en la importancia de que los materiales con los que los niños trabajan en la escuela sean realmente significativos. Cuatro aspectos, desde la perspectiva de estos autores, contribuyen a configurar una "escritura auténtica».

En primer lugar, el tipo de información que se maneja. Muchas veces, el contenido que los niños introducen en los escritos propuestos por el maestro tiene que ver muy poco con lo que realmente ocurre en su vida cotidiana e, incluso, con sus intereses. Ello contribuye a que la información que se trasmite sea, en muchos casos, muy similar entre todos los niños de la clase, incluso cuando sus experiencias tienen poco que ver entre sí. Por el contrario, cuando escriben espontáneamente el contenido es mucho más personal y existen importantes diferencias entre los niños.

En segundo lugar, la atención que los niños ponen en su trabajo, incluso la forma en que llegan a tener presente la audiencia, varía también de unas situaciones a otras. Ello se observa, por ejemplo, en la forma en que van evolucionando sus escritos cuando trabajando en clase sobre sus propios relatos, se van dando cuenta de que éstos serán leídos e interpretados por niños. Por ejemplo, se preocupan de que sus ilustraciones sean cada vez más cuidadas y de que estén en relación con la historia, incluso los niños pueden trabajar en diferentes borradores o preguntan al maestro en qué momento la historia puede considerarse finalizada.

Hay que tener también en cuenta el grado de integración con que se presenta la información para diferenciar la escritura auténtica de la que no lo es. En el aula ello se observa, sobre todo, en los escritos de los niños cuya única audiencia esperada es el maestro. Cuando exponen contenidos escolares esperando, simplemente, que éstos sean evaluados por el profesor, su grado de integración es mucho menor. Por el contrario, cuando los niños producen un texto de contenido científico, al que consideran algo más que un mero instrumento para que sus conocimientos sean evaluados por el profesor, el texto adquiere características específicas: los niños introducen descripciones de lo que han observado, además, son capaces de relacionarlo con aquello que han leído. explican lo que ven y predicen cambios futuros. Incluso se enfrentan de otra manera a los comentarios que introduce el profesor, éstos les ayudarán a mejorar su escrito. En suma, lo importante es que la escritura adquiere un nuevo sentido cuando se integra con el resto de las actividades de currículum.

Otro aspecto importante a tener en cuanta en los escritos infantiles son las metas que se propone lograr el escritor. Estas pueden ser muy variadas. Por ejemplo, a veces escriben para distraer a los otros, como ocurre cuando preparan sus cuentos para ser editados y formar parte de las actividades de la clase, otras componen textos para transmitir información a sus compañeros como ocurre cuando elaboran un texto expositivo o descriptivo en clase de ciencias naturales. Podríamos citar multitud de ejemplos. En suma, la meta que se pretende lograr es un aspecto de gran importancia para lograr una escritura "auténtica» a la que tanto el escritor como el lector le encuentren un significado. 


\section{ALGUNAS ACTIVIDADES PARA ACERCARSE A UNA ESCRITURA SIGNIFICATIVA}

Hasta ahora nos hemos fijado en la idea de «escritura auténtica» o, en cierto sentido, también significativa. El reto que, sin duda ninguna, el educador tiene ante sí será ayudar a los niños a producirla. Antes de sugerir algunas actividades concretas podemos señalar los principios que estarían tras la filosofía que propone el movimiento del lenguaje integrado. A ellos se refiere Hairston (1982) cuando distingue entre una aproximación tradicional a la enseñanza de la escritura y lo que podrían considerarse "paradigmas emergentes». A esto últimos pertenece el lenguaje integrado y sus rasgos aparecen en la tabla $I$.

\section{TABLA I}

Introducir la escritura en el aula: el enfoque del lenguaje integrado

(a partir de Hairston, 1982)

- Se presta especial atención al proceso más que al producto, es en él donde interviene el profesor

- Se enseñan estrategias de invención y descubrimiento. El profesor ayuda al estudiante a generar el contenido y a descubrir sus propias metas

- El profesor evalúa el producto en función de las intenciones del escritor y del grado en que se adapta a la audiencia

- La escritura se ve como un proceso recursivo más que lineal, la pre-escritura, escritura y revisión son actividades que se entretejen y solapan

- Se trata de un proceso global, la escritura incluye aspectos que van más allá de la racionalidad

- Esta aproximación pone el acento en su aprendizaje y desarrollo tanto como en las habilidades de comunicación

- Se incluyen múltiples tipos de textos, expositivos, expresivos, etc.

- La escritura se considera una actividad creativa que puede ser analizada y descrita. Se piensa que la escritura puede enseñarse

- Se piensa que quien enseña a escribir debe ser alguien que escribe. Los niños necesitan estar ante un experto que produce textos, que produce también errores y que necesita escribir varios borradores

Nos fijaremos ahora en algunas de las actividades que quienes trabajan desde la perspectiva del lenguaje integrado han propuesto para facilitar en el niño la adquisición de «una escritura» auténtica y significativa.

\section{La escritura expresiva}

La escritura expresiva es la que se utiliza a diario, puede considerarse como un medio de exploración personal y en la que están presentes los intentos de dar forma a las ideas que surgen en la conversación. Se utiliza para expresar ideas próximas a la persona, sin las limitaciones de las convenciones que están presentes en algunos formatos textuales específicos, como por ejemplo la descripción o la exposición. La práctica de la escritura libre puede considerarse un medio para generar y expresar ideas. Tchudi (1987) cree que el maestro debe ayudar al niño a encontrar una voz en sus escritos antes de llevarle a comunicarse con otras audiencias. Encontró que es útil para los niños el formato de carta privada como vehículo para expresar sus propios sentimientos y valores en un amplio rango de experiencias. Esas cartas son una 
muestra del valor del lenguaje para clarificar la experiencia y trabajar a través de los sentimientos. En este contexto quizás los niños no necesitan otra respuesta del maestro que su actitud de escucha. Este autor mostró también que, en ocasiones, el hecho de poner excesivo énfasis en presentar y publicar la propia producción a una amplia audiencia puede generar en el niño una excesiva presión que le impide centrarse en aquello que realmente quiere expresar.

\section{Las cartas}

Son un excelente instrumento de comunicación que, además, permiten al maestro observar el progreso que tiene lugar durante una determinada etapa escolar. Esto es lo que muestra uno de los trabajos de Newman (1985c) dedicados a esta cuestión. La autora, durante un periodo que pasa fuera de la escuela, intercambia cartas con una de sus alumnas y observa los progresos que en la niña se producen durante esa etapa. Nos aporta algunas reflexiones que resultan interesantes para captar la evolución en la escritura de la niña: en principio, toma la decisión de escribir algo que tenga significado para ella, con independencia de que conozca o no la ortografía correcta de las palabras. La niña muestra, incluso en sus primera cartas, que sabe algo sobre este tipo de textos, por ejemplo, que las cartas tienen diversos componentes: inicio, cuerpo, despedida. En cartas sucesivas se van observando nuevos progresos. Va tomando riesgos en su escritos. El aprendizaje, por otra parte, se produce en diversos frentes: coordinación de las ideas, uso de convenciones, atención a la audiencia, etc. En suma, la cartas aportan a los niños oportunidades para comunicarse entre ellos o con los adultos.

\section{El diario}

Estamos también ante un instrumento mediante el cual los niños utilizan el lenguaje escrito para comunicarse con otros niños o con el maestro en el contexto del aula (Fulwiler, 1987; Sanford, 1988; Unia, 1985). Cuando se utiliza, la meta fundamental del escritor es expresar y fijar ideas. En él los aspectos mecánicos del lenguaje como la ortografía, la puntuación, etc. pasan a un segundo plano y adquieren importancia las funciones comunicativas que se hacen presentes de forma más fácil cuando la evaluación como tal pasa a un segundo plano. Se trata de un vehículo de expresión que no va a ser corregido. Los niños pueden incluir en él aspectos tan variados como lo que han aprendido en una determinada clase, lo que han hecho el fin de semana $o$ aquello que, en determinadas ocasiones, quieren comunicar a su maestra. El profesor puede responderles, aunque ello no ocurra siempre. Muchas veces son suficientes unas breves palabras para mantener la comunicación. Esas respuestas cortas pueden tener un efecto importante en la escritura de los niños. La meta del maestro, en muchas ocasiones, no es mostrar las convenciones del lenguaje sino aportar al niño la impresión de aceptación de su trabajo y animarle en el proceso de comunicación. En estos casos el papel del maestro se asocia, simplemente, al hecho de ser un facilitador.

Especialmente interesantes son «los diarios de aprendizaje». Entre sus funciones cabe destacar las siguientes: a) el niño, mientras escribe, puede reflexionar sobre su aprendizaje y los contenidos del currículum; b) permite establecer conexiones entre los contenidos que aprenden en clase y sus propias creencias y valores; c) anima a los alumnos a articular conceptos curriculares en su propio lenguaje. Una particularidad de este tipo de diarios es, por ejemplo, "el libro de lecturas", donde los niños anotan 
comentarios sobre los libros leídos y aportan opiniones personales sobre los personajes, sucesos, temas y valores que aparecen en ellos.

Numerosos autores se refieren a sus experiencias concretas en las aulas cuando utilizan el diario de clase. Crocker (1982) señala que utilizó diarios para establecer el diálogo con sus alumnos de sexto curso de la escuela elemental. Los niños particịpaban en sesiones semanales para escribir el diario, se trataba de una actividad que habían de realizar en el aula y que había sido previamente programada. Escribían también de forma individual cuando ellos quieren. El maestro recogía los diarios una vez a la semana y comentaba las ideas de los niños sin que ello significara una evaluación formal.

Anderson (1992) se refiere a las ventajas e inconvenientes de urilizar diarios incluso en cursos con adultos, por ejemplo en la formación de profesores. En este caso no todo son ventajas. Entre lo problemas que presentan cita los siguientes: a) tal vez en los cursos en los que participan adultos se ha producido un uso excesivo del diario de forma que, en ocasiones, puede crear ciertas confusiones; b) algunos estudiantes abusan de su informalidad y reflejan sus propios prejuicios en temas que resultan excesivamente polémicos, incluso para atacarse entre sí; c) a algunos estudiantes no les resulta una actividad agradable $\mathrm{o}$, sobre todo, les disgusta que el hecho de escribir el diario se convierta en una obligación; d) evaluar los diarios puede resultar difícil para el profesor, en este sentido se insiste en que muchas veces resulta difícil para los alumnos reflexionar sobre su propia práctica; e) a veces resulta difícil para los alumnos escribir el diario y éste se convierte en un conjunto de apartados que tienen escasa conexión entre sí, otras veces los diarios son en un simple escrito para el profesor.

Algunas reflexiones de Anderson (1992) tratan de superar estos problemas. En primer lugar, tal vez el diario sea una estrategia de aprendizaje entre otras y es necesario reflexionar sobre el modo en que puede integrarse en el conjunto del proceso. En segundo lugar, habrá que pensar también que quizás pueda considerarse una forma de facilitar la reflexión sobre la propia práctica que no da el mismo resultado con todos los estudiantes, será necesario considerar la posibilidad de que el diario sea utilizado solamente por quienes voluntariamente se presten a ello. Por último, hay que replantear el modo en que se lleva a cabo su evaluación.

\section{El taller de escritura}

Quienes adoptan la perspectiva del lenguaje integrado saben bien que en la aulas los niños escriben con diferentes metas en distintos momento del día. Sin embargo puede ser útil, para favorecer el aprendizaje del niño, que el profesor programe también un «taller de escritura». Los niños trabajan tanto individualmente como en pequeños grupos, incluso compartiendo sus borradores y ayudándose entre ellos. El papel el maestro puede variar, orientando su atención a los grupos, a la producción individual, etc. Quizás la característica más notable de estos talleres sea su flexibilidad, ya que se adaptan a una gran variedad de actividades.

Entre los materiales del trabajo del taller las carpetas pueden desempeñar un papel importante y si nos detenemos un momento ante ellas prestaremos atención al modo en que se organizan. Por ejemplo los niños pueden ir manteniendo los diversos borradores, el trabajo en curso y las actividades finalizadas. Pueden incluirse también orientaciones para una evaluación personalizada, por ejemplo mediante una lista con las habilidades que el niño deberá mostrar en sus escritos.

En el taller de escritura ocupan un lugar importante las interacciones con el maestro y con los compañeros. La meta de dichas interacciones puede ser ayudar a los niños a generar u organizar ideas, identificar la audiencia o planificar como va a presentarse 
y publicarse. La relación con el profesor, permite también al maestro valorar el progreso del niño. Graves (1983) presenta seis principios de apoyo a niño, por parte del maestro, que ayudarán a que esas interacciones sean útiles.

- En la interacción han de existir aspectos predecibles, es decir, el niño ha de estar seguro de que aunque tome ciertos riegos en su escritura el profesor le proporcionará ayudas que le permitan progresar.

- La interacción ha de centrarse en determinados aspectos específicos, por ejemplo, cuál es la meta del escritor y cómo va a lograrla.

- El niño ha de poder esperar soluciones demostrables ante sus problemas de escritura. No es productivo hablar de forma excesivamente general, sino aspectos concretos; por ejemplo, mostrar con ayuda de un retroproyector cómo podrían variarse determinadas frases.

- El niño debe tener la libertad de plantear cuestiones, incluso ser él mismo quien organiza la interacción.

- Puede ser útil el uso de nomenclaturas en relación con aspectos relacionados con el proceso o elementos técnicos de la escritura.

- La interacción ha de ser algo atractivo para el niño. Incluso el humor puede contribuir a ello.

También pueden ser útiles las situaciones de interacción entre los iguales. en este caso los niños pueden aportar observaciones y comentarios, incluso por escrito, a las producciones de sus compañeros. Por ejemplo, el trabajo de un niño puede circular entre los participantes de un grupo pequeño que, en hoja parte, le aportarán sus sugerencias. Es interesante que el maestro entrene a los niños a ser «tutores de sus compañeros». Calkins (1983) diferencia en estas relaciones con los iguales dos tipos: formales, que son organizadas por el maestro, e informales, que son iniciada por los propio niños. Lo que considera esencial es que en la clase se produzca un clima que las favorezca. Dos aspectos cabe resaltar: a) escribir en grupo aporta a los niños oportunidades para hablar sobre sus metas y las dificultades que tienen para lograrlas; b) les ayuda a descubrir el efecto que su escrito tiene sobre los lectores.

\section{ESCRIBIR A TRAVÉS DEL CURRÍCULUM}

Hemos repetido en numerosas ocasiones que la lecto-escritura, desde el enfoque del lenguaje integrado, ha de ser algo significativo tanto para el escritor como para el lector. El lenguaje ha de tener metas prácticas y no se trata sólo de algo que haya de ser aprendido como una abstracción. En la escuela el aprendizaje del lenguaje no puede ser ajeno al que se produce en el conjunto de las actividades del currículum.

\section{Escribir en clase de literatura}

¿Qué diferencias existen entre la aproximación que ofrece el lenguaje integrado y la forma en la que los niños se acercan a la literatura desde un enfoque tradicional? Tal vez la meta fundamenta sea lograr que los niños se aproximen al texto de una forma activa, creando además la posibilidad de que surja ante él un amplio número de interpretaciones. Para lograrla Butler (1991) sugiere las actividades que se incluyen en la tabla II. 


\section{2}

\section{TABLA II}

Actividades en clase de literatura

(a partir de Butler, 1991)

\begin{tabular}{ll}
\hline \multicolumn{1}{c}{ ACTIVIDADES } & \multicolumn{1}{c}{ INTERÉS Y JUSTIFICACIÓN } \\
\hline Hacer un cartel para anunciar un libro & $\begin{array}{l}\text { Permite combinar la expresión gráfica y la } \\
\text { palabra para captar el aspecto de mayor } \\
\text { interés }\end{array}$ \\
Fichero de lecturas recomendadas & $\begin{array}{l}\text { Cada niño incluye, además de los datos del } \\
\text { libro, un pequeño comentario que oriente a } \\
\text { los demás }\end{array}$ \\
Cartas & $\begin{array}{l}\text { Al autor o al bibliotecario justificando por- } \\
\text { qué puede adquirirse un libro, cambiarlo de } \\
\text { sitio, etc. }\end{array}$ \\
Adaptación teatral & $\begin{array}{l}\text { Teniendo en cuenta los personajes y escenas } \\
\text { más importantes del libro los niños pueden } \\
\text { adaptar su contenido para representarlo }\end{array}$ \\
Rastrear los temas más importantes & $\begin{array}{l}\text { Ello les ayudará a realizar un informe de } \\
\text { clase. }\end{array}$ \\
\hline
\end{tabular}

\section{Escribir en clase de ciencias sociales}

Los textos que se proponen a los niños como tarea en estas clases son, sobre todo, de carácter imaginativo. Se asume que ello les ayudará a salvar la distancia cronológica o geográfica entre el periodo que estudian y su situación actual. Podrían proponerse, a título de ejemplo, tres tipos de actividades: a) dramatizaciones, presentaciones orales, debates y discusiones, combinadas con notas o planes que los niños realizarán por escrito; b) diarios personales en los que ellos mismos se sitúen en el lugar del personaje histórico que estudian; c) debates sobre problemas sociales que necesitan hacer presentes habilidades relacionadas con la argumentación.

\section{Escribir en clase de matemáticas}

Brown (1991) señala algunos aspectos que pueden servir de punto de partida a la enseñanza de la matemática desde el enfoque del lenguaje integrado. Se trata, en primer lugar, de eliminar algunos mitos que están presentes en las aulas y entre ellos se señalan tres: a) una idea modular del pensamiento, de acuerdo con la cual los niños aprenderían mediante procesos lineales que suponen la adquisición de una mera suma de habilidades cognitivas independientes de cualquier contexto; b) concentrarse en la «respuesta correcta", lo cuál favorecería un aprendizaje memorístico más que significativo; $c$ ) aceptar la sucesión de estadios fijos en el aprendizaje, con independencia de la tarea o del contenido aprendido. Frente a esta idea los autores proponen una aproximación que pone el acento en «las situaciones de resolución de problemas reales y significativos». Interesa centrarse en conceptos relevantes y de forma que tenga sentido para el niño. 
Aceptando este enfoque alternativo, la escritura puede desempeñar un papel importante. Wason-Ellam (1987), por ejemplo, realiza una investigación en la que alumnos de primer año de la escuela elemental responden una vez a la semana, en su cuaderno, a la siguiente cuestión: ¿Qué descubriste esta semana en el rincón de matemáticas? En esta clase los niños trabajan en diferentes rincones y, concretamente en el de matemáticas, llevaban a cabo experiencias manipulativas. Los comentarios de los niños muestran cómo crean su propio lenguaje interactuando con experiencias matemáticas. Un buen ejemplo de la utilidad del diario de escritura lo ofrecen los siguientes comentarios que uno de los niños anotó en él:

«Sabes como aprendí a sumar? Utilicé cosas viejas. Cogí primero unas cuantas cosa, después algunas más y las junté. El número que había era mayor que antes. Ahora lo sigo haciendo igual" (citado en Butler, 1991, p. 129)

En suma, la escritura introducida de este modo ayuda a los niños a reflexionar sobre su propio aprendizaje. Les ayudará, además, a explorar las relaciones entre lo que aprenden y sus experiencias previas.

\section{Escribir en clases de ciencias físicas y naturales}

La escritura en estas clases adquiere también características peculiares. La naturaleza de las materias permite que los niños lleven a cabo pequeñas investigaciones. Para ello habrán de anotar sus observaciones, leer determinados los materiales que les permitan obtener nueva información, escribir su propio informe, etc. También aquí lo importante es que los niños se dan cuenta de que su lenguaje es significativo para ellos mismos y para los demás. Newman (1983), por ejemplo, se refiere a las actividades de un niño de ocho años que utiliza su propio lenguaje para anotar sus observaciones sobre la conducta de las larvas de los gusanos. Sus dibujos y comentarios son la base de un libro que publicó mostrando a la vez sus habilidades como científico y como escritor. Anotaba, analizaba y predecía lo que las larvas harían en ciẹrtas condiciones. En el libro añadió también nueva información y reorganizó la que procedía de sus observaciones, de este modo llevó a cabo un proyecto muy similar al que experimentan los autores adultos. La lectura y la escritura vuelven a estar presentes en las aulas con metas reales.

\section{INTRODUCIR AL NIÑO EN LA LECTURA}

¿Qué significa realmente aprender a leer desde la perspectiva del lenguaje integrado? No cabe duda de que sus defensores se proponen ante todo una lectura comprensiva. La verdadera lectura comienza con la búsqueda del significado y se trata, por tanto, de un proceso que en ningún caso puede reducirse a una acrividad de decodificación gráfico-fonética. Es decir, leer es mucho más que asociar las letras, agrupadas en sílabas o palabras, a sus correspondientes sonidos. No hay duda de que nos hallamos ante una actividad compleja en la que el individuo se compromete en la búsqueda de significados. Desde esta perspectiva, las actividades implícitas en la lectura pueden situarse a través de un continuo. En su nivel más bajo aparecen destrezas superficiales de decodificación, son las que están presentes cuando el lector lee automáticamente sin estar atento al contenido del texto En los niveles intermedios de este continuo se accede al reconocimiento del significado de palabras, complementos, predicados aislados y oraciones simples. En el nivel superior se genera conscien- 
temente una representación, marco o modelo que, incluyendo elementos del conocimiento previo, permite entender, más allá de las palabras, el significado del texto.

¿Cómo aproximar a los niños a la lectura? Newman (1985b) en su trabajo aporta sugerencias de enorme interés. Son aspectos prácticos de este movimiento que, en alguna medida, se refieren a sus presupuestos. Podemos partir de un ejemplo. Teresa tiene tres años, su padre le lee todas las noches un cuento, a ella le gusta mucho y ha llegado a aprenderlo casi de memoria. Un día lo muestra a su abuela, en presencia de su padre. Comienzan a leerlo juntas. La niña no sabe realmente leer, o mejor, no sabe decodificar cualquier texto del modo en que lo haría un adulto. Sería difícil para ella acercarse a materiales que no tienen un significado, pero su cuento es realmente significativo. El adulto comienza a leer la primera palabra con enorme entonación, se para y la niña, mirando al libro de la forma como lo haría un lector experto, continua, y «recita el cuento». Un observador ajeno podría pensar que «sabe realmente leer». La niña va narrando el cuento y, en ocasiones, introduce nueva información que ella misma aporta. Cuando se detiene,su abuela lee una o dos palabras que le ayudan a seguir. De este modo van «leyendo juntas» hasta que el cuento finaliza. Este ejemplo nos permite comprender un posible camino para acercar a los niños al mundo de la lectura. En primer lugar, leer no significa atenerse sólo a lo que aparece impreso y se muestra como un estímulo visual, los niños han de interpretar lo que leen. Para dar sentido a lo leído el niño actualiza aquello que sabe acerca del funcionamiento del lenguaje, incluso de cómo lo impreso se relaciona con el lenguaje oral y, de este modo, va encontrando el sentido de lo que lee. La lectura supone la interacción entre diversas fuentes de información que el lector debe coordinar. Los niños occidentales están en contacto, en la escuela y fuera de ella, con múltiples fuentes impresas que les permiten actualizar lo que saben sobre el lenguaje, organización de historias u otros conocimientos que les ayudan a interpretar los materiales impresos.

Esta interacción de Teresa con su abuela es un buen ejemplo de cómo la lectura puede entenderse un proceso que va mucho más allá de la simple decodificación de las sílabas, es decir, de un proceso por el cual el niño aprende a establecer relaciones entre el grafismo y el sonido. Se trata, sobre todo, de acercar al niño a una lectura significativa. Desde esta perspectiva, leer con los niños en situaciones de la vida cotidiana, que transcienden el mundo de la escuela, tiene importantes funciones. En primer lugar les muestra cómo el lector se compromete en el proceso de forma fluida; les acerca, además, a aspectos "casi materiales", por ejemplo, al modo en que se manejan los libros o a como se vuelven las páginas, de forma que se den cuenta de que lo escrito no finaliza sino que continua. Aprenden también aspectos relacionados con las estructuras linguísticas, por ejemplo el modo en que se construyen las historias, cómo se introducen los personajes o se suceden los acontecimientos, también cuales son las relaciones entre ellos y como van cambiando los personajes como consecuencia de lo que allí ocurre. Es importante, por otra parte, no sólo acercarles a las historias, sino también a otro tipo de materiales impresos con los que se han de encontrar en el futuro. De ese modo irán comprendiendo que es posible aprender muchas cosas a través de los libros. En suma, leer con los niños es una actividad que les permite incorporar muchas de las sutilizas de los lectores sin tener que realizar el proceso en su totalidad. Entre todas esas sutilizas existe, quizás, una realmente importante: el niño ha de aprender qué hacer cuando aquello que lee no tiene realmente sentido; en este punto son posibles varias alternativas: esperar a que la próxima información les ayude a comprenderel significado, leer de nuevo buscando algún aspecto especialmente relevante ante la dificultad, buscar aspectos relacionados con lo que se está leyendo. Cuando se corrigen los errores de los niños será necesario hacer presentes estas estrategias. 


\section{UNA REACCIÓN A LOS PROGRAMAS BASICOS}

Newman (1985d) narra una de sus experiencias relacionadas con la lectoescritura que nos parece interesante citar en este punto. En un curso sobre lectura la autora pidió a los maestros que describieran sus estrategias cuando, ante un texto, aparecía algo que no comprendían. Entre su lista de respuestas se encontraban estrategias como las siguientes: evitaban lo que desconocían, se acercaban a través del contexto, lo sustituían por palabras o frases que parecían mantener el significado, volvían a leer el pasaje o repasaban lo anterior para encontrar información que contribuyera a clarificar la dificultad. Estos maestros describieron también las estrategias que ellos mismos sugerían a los niños cuando éstos tenían dificultad en la lectura: se preocupaban de que los niños identificaran correctamente las palabras, incluso les indicaban que se fijaran en los sufijos y prefijos, $o$ incluso que prestaran atención a las palabras que no conocían. En suma, orientaban a los niños a lo desconocido. Tras estas estrategias de los enseñantes podríamos descubrir sus creencias relacionadas con la idea de que de que el significado depende de la identificación de palabras específicas, en el caso de que éstas no se comprendían no se captará el sentido del texto.

Esta dicotomía entre el modo en que los maestros enseñan a los niños o aprenden ellos mismos puede ser una ilustración acerca de dos puntos de vista presentes en la enseñanza de la lectura en los últimos años. Un punto de vista tradicional, inspirado quizás en los programas básicos, que considera a la lectura como un proceso de relación pasiva entre el lector y el mundo impreso; es decir, la información necesaria para leer reside sobre todo en la página y fluye desde allí al lector. De acuerdo con esta perspectiva, la lectura supone una serie de pasos que comienzan con la identificación visual de las letras y las palabras y está seguida por el establecimiento de formas visuales y sus correspondientes sonidos para producir la expresión oral, a partir de la cual se obtiene el significado. En contraste con este enfoque existe un punto de vista alternativo que considera a la lectura como algo más que un proceso fundamentalmente visual. Por el contrario, se piensa que el lector ha de manejar información que procede de más de una fuente. La lectura supone un intercambio entre el medio impreso y el conocimiento aportado por el lector que se refiere, por ejemplo, a cómo ha de orientar la mirada a la página, a la estructura y flujo del lenguaje, al conocimiento específico del tema y del mundo en general, etc. En suma, el lector necesita aportar considerable información cuando se compromete con el lenguaje escrito y tiene que descifrarlo.

Pero, ¿qué son realmente los programas básicos a los que la filosofía del lenguaje integrado se presenta como una reacción? Se trata de paquetes didácticos, que contienen un material seleccionado y secuenciado por niveles orientado a desarrollar en los niños habilidades específicas (Anderson $e t$ al., 1985). Su aplicación incluye el uso de una serie de estrategias instruccionales para acercar al niño a la lecto-escritura que han tenido una enorme influencia en la forma en que muchos profesores han enseñado a los niños a leer. No creemos exagerado decir que quizás más del ochenta por ciento del tiempo que se dedica en las aulas a enseñar a los niños a leer y escribir se realiza de acuerdo con la metodología de estos programas.

Sus características serían las siguientes:

- Aíslan los sonidos, letras y palabras del sistema del lenguaje para extraer actividades en las que se practiquen habilidades aisladas.

- Se pierden los significados contextuales, produciendo abstracciones distorsionadas.

- En el proceso de controlar el vocabulario y la sintaxis se pierde el estilo personal del escritor. 
- El material natural de lectura al que los niños tienen acceso cotidianamente es radicalmente distinto al que ven en el contexto del aula.

- El niño no tiene posibilidades de acceder a materiales que realmente le interesen y que le sirvan de apoyo en el proceso de aprendizaje.

En los programas basales, por otra parte, aprender el lenguaje en forma de memorización de reglas y prácticas de formas centradas en no cometer errores implica un esfuerzo y ansiedad que no se dan en el aprendizaje en ambientes naturales. En estos últimos el lenguaje se adquiere de forma natural en la interacción con otros. El esfuerzo mental por evitar los errores cede paso a la fluencia y al dominio práctico, algo que hará más fácil el progreso.

\section{LA ALTERNATIVA DEL LENGUAJE INTEGRADO}

¿Qué se entiende, desde el enfoque del lenguaje integrado por un aprendizaje natural de la lectura? Seguramente el lector que haya tenido la paciencia de seguirnos hasta aquí lo habrá comprendido ya, pero en cualquier caso intentaremos especificarlo. Su punto de partida está en la idea de que las habilidades lectoras son adquiridas de la misma manera en que aprendemos a hablar. La descripción de lo que quienes se sitúan en esta perspectiva consideran un aprendizaje significativo podría ser tomada, en un principio, como una analogía útil para reproducir en la clase condiciones naturales y significativas algo menos articuladas, artificiales y directivas de las que se han empleado tradicionalmente en el aprendizaje de la lecto-escritura. Sin embargo, a lo que parecía ser una analogía práctica entre las condiciones de aprendizaje del lenguaje oral y escrito, se le ha dado un estatus teórico de identidad. Es decir, ya no se trata de que el lenguaje oral y escrito se puedan adquirir en condiciones o situaciones de aprendizaje similares, sino de que ambos comparten los mismos procesos. Los problemas que lleva consigo asumir este supuesto ya han sido planteados en un artículo anterior incluido en esta monografía.

Buscando las condiciones que, en su opinión, se esconden tras el aprendizaje natural del lenguaje con el fin de encontrar en ellos el fundamento de sus propios programas para la enseñanza de la lecto-escritura, los autores que aceptan la perspectiva del lenguaje integrado han acudido a los trabajos que se fijan en cómo los niños adquieren el lenguaje oral. Se detienen, sobre todo, en cómo lo van aprendiendo de forma natural. MacGowan-Gilhooly (1991) insiste, por ejemplo, en la necesidad de participar de las conversaciones, escuchar y experimentar con la propia lengua, aprender primero el nombre de cosas, a continuación ir construyendo frases y, por último, utilizar la sintaxis que necesitan para expresarse ellos mismos. A partir de estas observaciones se extraen unos principios con los que se pretende fundamentar la práctica del lenguaje integrado en la enseñanza de la lectura:

- La enseñanza de la lengua debe estar centrada en el aprendiz.

- La lengua se aprende mejor cuando se mantiene íntegra y no se descompone en componentes ni habilidades específicas.

- La enseñanza de la lengua debe integrar la escucha, habla, lectura y escritura.

- El lenguaje ha de ser introducido en las clases de forma significativa y funcional.

- Situar a los niños en contextos sociales, en los que se crean contextos comunicativos, es de gran importancia en la enseñanza de la lengua.

En general, se progresa en la adquisición de la lectura a partir de interacciones plenas y masivas con padres, otros adulto o pares más capaces que facilitan el control que los niños pueden llegar a realizar del propio aprendizaje. 
Entre los principios en que se apoyan sus estrategias de enseñanza cabe destacar los siguientes.

a) Los adultos se consideran como modelos lectores. Podemos recordar, por ejemplo, la forma en que Teresa se acercaba a la lectura interactuando con miembros más expertos de su familia. La niña aprendió como un lector experto llega a comportarse ante el texto: por ejemplo, cómo coger el libro, la entonación de las frases, incluso la estructura de la historia, etc.

b) La lectura es valorada por encima de otras actividades en la vida cotidiana de los niños. El interés que los adultos, miembros más expertos de la comunidad, muestren por las situaciones y los materiales de lectura son factores decisivos no sólo para facilitar el aprendizaje infantil sino también para desarrollar actitudes positivas y naturales hacia la lectura.

c) La presencia de materiales de lectura significativos para el niño en sus actividades cotidianas y que no han sido elaborados con finalidades exclusivamente didáctica. Es decir, textos cuyo lenguaje no se aleje del lenguaje oral natural. Además, que el niño seleccione sus propios materiales fomenta que la motivación y el control vayan de la mano en el aprendizaje. En este contexto, algunos autores comentan que es importante que las historias con las que se encuentren los principiantes se amolden a su conocimiento implícito de la estructura de la historia, y ello porque la predicción puede facilitar ese aprendizaje en sus momentos iniciales. Parece sugerente, en este sentido, la posibilidad de registrar en formato escrito historias que el mismo niño cuente para que se conviertan en material de trabajo para el mismo niño o sus compañeros de clase.

d) El aprendizaje de la lengua en situaciones naturales se centra en la negociación de significados y no en la corrección de la forma. Está guiado por los intereses del niño y libre de ansiedad, de aquí que una aproximación afectiva al aprendizaje de la lectura se considere importante. Un ambiente natural cargado de elementos motivadores les acerca a la lectura como una actividad reconfortante que les aporta cosas, y ello lejos de la ansiedad por adecuarse a unos criterios de evaluación que continuamente exigen al niño logros inmediatos.

En suma, enseñar a leer adoptando la filosofía del lenguaje integrado supone introducir a los niños en el medio impreso como algo significativo y tomando como punto de partida el contexto en el que cotidianamente se desenvuelven.

\section{CONCLUSIONES} clusión:

Algunas reflexiones que intentan sintetizar lo expuesto nos servirán de con-

1) Los niños, cuando llegan a la escuela saben más del mundo primero de lo que a primera vista pudiera penarse.

2) Los conocimientos infantiles son inseparables del contexto social en el que los niños se desenvuelven, especialmente de sus interacciones más tempranas en la familia con el medio impreso. Los padres, los adultos en general y otros compañeros crean diversos entornos cotidianos en los que los niños se desenvuelven y donde se llevan a cabo actividades relacionadas con la lecto-escritura.

3) Para quienes aceptan el enfoque del lenguaje integrado, la escucha, la palabra, la lectura y la escritura son actividades entre las que existe una profunda interacción. Por ello, de acuerdo con estos autores, a enseñanza y el aprendizaje de la lengua no puede producirse poniendo el acento en una de ellas y con independencia de las demás. 
4) Por lo que se refiere a la enseñanza de la escritura, el enfoque del lenguaje integrado surge como una alternativa a los programas tradicionales en los que se concede más importancia a la forma que a la función de la lengua y en la que los aspectos mecánicos ocupan un lugar central.

5) Los enseñantes buscan aproximar al niño a una "escritura auténtica», para lograrlo será necesario tener en cuenta: el tipo de información que se maneja, la necesidad de prestar atención a la audiencia, el grado de integración con el que se presenta la información y las metas del escritor.

6) Para que los niños lleguen a producir una «escritura auténtica» los profesores proponen las más diversas actividades: cartas, diarios, talleres de escritura, etc. El principio en que todas ellas se apoyan propone que los niños «escriben para aprender» y no tanto que "aprenden a escribir».

7) De un modo similar, la enseñanza de la lectura se orienta sobre todo a la búsqueda del significado. Leer no puede reducirse a un proceso de decodificación gráfico-fonético. Los niños aprenden a leer aportando para ello todo un conjunto de conocimientos previos que les ayudan a descubrir el sentido de lo impreso.

8) Los programas de enseñanza de la lectura que se inspiran en la filosofía de lenguaje integrado surgen también como reacción ante otro más tradicionales, en este caso los "programas basales", paquetes didácticos orientados sobre todo al desarrollo de habilidades específicas y parciales.

9) En suma, enseñar a leer y escribir, adoptando la filosofía del lenguaje integrado, exige que os niños se acerquen al medio impreso como algo significativo. Los aspectos comunicativos y funcionales del lenguaje pasan a primer plano.

10) Si bien las aportaciones de este movimiento en el terreno de la práctica pueden ser de gran interés, hemos de reconocer que e necesario un esfuerzo de revisión de sus presupuestos teóricos.

\section{Referencias}

ANDERSON, J. (1992). Journal writing: The promise and the reality. Joumal of Reading, 36(4), 304-309.

ANDERSON, R. C., et al. (1985). Becoming a nation of readers: The report of the commssion on reading. Washington, DC: U.S. Department of Education.

ATWELL, N. (1987). In the Middle. Exeter: Heinemann.

BRowN, C. L. (1991). Whole concept mathematics: A whole language application. Educational Horizons, 69(3), 159-163.

Butler, S. (1991). The writing connection. En V. Froese (Eds.), Whole-language. Practice into theory (97148). Boston and London: Allyn and Bacon.

CALKINS, L. (1983). Lessons from a child. Portsmouth, N. H.: Heinemann.

CLAY, M. (1975). What did I urite? Londres: Heinemann.

CroCKer, M. (1982). Dialogue journals promote interpersonal relationships. Highway One: Canadian Journal of Language Education, 5(3), 34-41.

EDELSKY, C., y SMITH, K. (1989). Is that writting-or are those marks just a figment of your curriculum? En G. Manning y M. Manning (Eds.), Whole language: Beliefs and practice, K-8 (183-193). Washington, DC: National education Association.

Fulwiler, T. (1987). Writing and learning, grade three. En T. Fulwiler (Eds.), The joumal books Portsmouth, NH: Boynton / Cook.

GRAVES, D. (1983). Writing: teachers and children at work (Trad. cast Didáctica de la escritura. Madrid: Morata-MEC, 1991). Portsmourh, NH: Heinemann.

HAIRSTON, M. (1982). The winds of change: Thomas Khun and the revolution in the teaching of writing. College Composition and Communication, 33(1), 76-88.

HaRsTE, J.; WoOdWARDD, V., and BurKe, C. (1984). Language stories and literacy lessons. Portsmouth, N. H.: Heinemann Educational Books.

MACGOWAN-GILHOOLY, A. (1991). Fluency first: reversing the traditional ESL sequence. Journal of Basic Writing, 10(1), 70-81.

NewMan, J. (1983). J. P. becomes an eight-year-old scientific writer. Heighway One: Canadian Journal of Language Education, $6(3), 48-58$. 
NEWMan, J. (1985a). Conferencing: Writing as a collaborative activity. En J. Newman (Eds.), Whole Language. Theory in use (123-130). Porstmouth, NH: Heinemann.

NEWMAN, J. (1985b). Insights from recent reading and writing research and their implications for developing whole language curriculum. En J. Newman (Eds.), Whole Language. Theory in use (pp. 736). Porstmouth, NH: Heinemann.

Newman, J. (1985c). To Judith: A look at a child's writing development through his letters. En J. Newman (Eds.), Whole Language. Theory in use (73-82). Porstmouth, NH: Heinemann.

NewMan, J. (1985d). Using children's books to teach reading. En J. Newman (Eds.), Whole Language. Theory in use (pp. 55-64). Porstmouth, NH: Heinemann.

SANFORD, B. (1988). Writing reflectively. Language Arts, 65(7), 652-657.

TCHUDI, S. (1987). Writer to reader to self: The personal uses of writing. Language Arts, 64(5), 489-496.

TUNNELL, M. O. (1989). Using real books: Research findings on literature based reading instruction. The reading Teacher, March, 470-477.

UNIA, S. (1985). From sunny days to green onions. En J. Newman (Eds.), Whole Language. Theory in use (pp. 65-72). Porstmouth, NH: Heinemann.

WASON-ELLAM, L. (1987). Writing across the curriculum. Canadian Journal of English Language Arts, $11(3), 5-23$

\section{Leer y escribir: ¿cómo lograrlo desde la perspectiva del lenguaje integrado?

$$
\begin{aligned}
& \text { Pilar Lacasa, José J. Anula y Beatriz Martín del Campo } \\
& \text { CL\&E, 1995, 25, pp. 31-49 }
\end{aligned}
$$

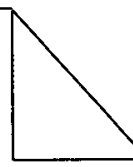

Resumen: El objetivo de este artículo es presentar, con cierto detalle, los presupuestos básicos y las prácticas educaticas que se aglutinan alrededor del «lenguaje integrado" cuando se trata de enseñar a los niños a leer y escribir. Tras profundizar en los procesos de lectura y escritura, tal como se entienden desde este enfoque, se revisan situaciones y actividades desde los que pueden introducirse en el aula.

Datos sobre el autor: Pilar Lacasa es Profesora Titular de Psicología Evolutiva y de la Educación. Actualmente trabaja en temas relacionados con el desarrollo, aprendizaje y enseñanza de la lecto-escritura desde un enfoque socio-cultural.

José Jerónimo Anula es Licenciado en Psicología por la Universidad de La Laguna. Actualmente es becario del Plan de Formación de Personal Docente e Investigador. Trabaja en el campo de las creencias y las actitudes y trata de acercarse a ellas a partir del análisis del discurso.

Beatriz Matín del Campo es Licenciada en Psicología por la Universidad Autónoma de Madrid. Actualmente es becaria del Plan de Formación de Personal Docente e Investigador de la U.N.E.D. Trabaja en temas relacionados con e desarrollo de la lengua escrita, le interesa especialmente el tema de los formatos textuales.

Agradecimientos: Gracias a Mónica, Enrique y Teresa que nos han permitido observar cómo aprender a leer $y$ escribir.

Dirección: Pilar Lacasa. Departamento de Educación. Facultad de Ciencias de la Educación. Universidad de Córdoba. Priego de Córdoba, n 4.14013 Córdoba. FAX: 957 218923. E-Mail: Pilar.Lacasa@uned.es.

José J. Anula y Beatriz Matín del Campo. Departamento de Psicología Evolutiva y de la Educación. Facultad de Psicología. U.N.E.D. Ciudad Universitaria s/n. 28040 Madrid. Fax: 91 3986216.

( $)$ PERMISOS PARA CITAR O REPRODUCIR EN OTRAS FUENTES: Se pueden citar libremente hasta 500 palabras. Para reproducir una porción de texto mayor, figuras o ilustraciones, se deberá pedir permiso por escrito a la revista, especificando el uso al que se destina el texto. En todos los casos, se deberá citar el copyright de CL\&E. En el caso de artículos o textos que hayan sido a su vez reproducidos en $C L \& E$ los interesados deberán dirigirse tanto a los detentadores del copyright original como a $C L \& E$, en el caso de que se quiera hacer uso de la traducción. FOTOCOPIAS: Para todo lo relacionado con el uso mediante fotocopia del material de esta revista, deberán dirigirse a: CEDRO, C/ José Marañón, 10, $3 .^{\circ}$ Izda. Tel. 5941575 . Fax 4453567 